Research Article

\title{
Neural Network-Based Laser Interferometer Compensation for Seismic Signal Detection
}

\author{
Kyunghyun Lee, Hyungkwan Kwon, and Kwanho You \\ Department of Electrical Engineering, Sungkyunkwan University, Suwon, Republic of Korea \\ Correspondence should be addressed to Kwanho You; khyou@skku.edu
}

Received 19 July 2017; Revised 18 September 2017; Accepted 4 October 2017; Published 22 February 2018

Academic Editor: Jesus Corres

Copyright $\odot 2018$ Kyunghyun Lee et al. This is an open access article distributed under the Creative Commons Attribution License, which permits unrestricted use, distribution, and reproduction in any medium, provided the original work is properly cited.

\begin{abstract}
We propose a seismic wave detection method in the frequency domain using a heterodyne laser interferometer, which is used in ultraprecision fields as a displacement measurement device. In seismology, it is important to accurately measure seismic waves. To overcome the limited frequency range and low resolution of accelerometers and velocimeters and to enhance the precision of seismic data analysis, we use the heterodyne laser interferometer as a seismic detection apparatus. We apply the data fusion algorithm with the adaptive standard deviation ratio ( $)$ derived from the neural network to improve the laser interferometer's measurement precision. Moreover, by using the interferometric characteristics, we analyze the seismic data in the frequency domain. To determine the location of the epicenter from the body wave propagation analysis, we apply the STA/LTA algorithm to the measurement data. The effectiveness of the proposed laser interferometric seismometer is shown through experiments to locate the precise epicenter.
\end{abstract}

\section{Introduction}

An earthquake is a devastating but common natural disaster around the world. To reduce and predict the damage caused by earthquakes, it is necessary to precisely measure and analyze the seismic signal. Hence, the study of earthquakes has been performed through various seismic analyses and predictions. Methods to analyze earthquakes include spectrum analysis, magnitude measurement, intensity interpretation, and epicenter decision. The short-time average/long-time average (STA/LTA) trigger algorithm is commonly applied in microseismic monitoring systems and has gained significant increased interest. In the STA/LTA algorithm, the most important factor for seismic wave analysis is to identify a seismic event that includes an external disturbance. Widely used seismic measurement devices include seismic velocimeters and accelerometers. These apparatuses detect seismic waves based on force and pressure sensors. They can be classified according to their measurable frequency range as short-period, long-period, and broadband seismographs.
These apparatuses have defects such as limited frequency range and low resolution. Therefore, errors exist when calculating the exact $\mathrm{P}$ - and S-wave arrival time.

To improve seismic analyses, Colak et al. [1] proposed a new P- and S-wave arrival time decision method. The discrete wavelet transform was used to analyze the change of frequency and energy in high frequency bands. As a result, $\mathrm{P}$ - and S-wave arrival time can be measured using a series of data windows. Hloupis and Vallianatos [2] suggested an earthquake early warning system, based on a redundant wavelet transform as a processing tool to rapidly estimate earthquake magnitude. The suggested algorithm strengthens the ability to distinguish body waves from noise. Panagiotakis et al. [3] proposed an automatic identification method of P-phase arrival based on energy and frequency characteristics of the local maxima distribution. Moreover, the method performs a robust detection against the background noise of seismograms. Baziw [4] proposed a robust seismic event identification algorithm by obtaining the optimal estimate of a possible seismic event based on the Rao-Blackwellized 


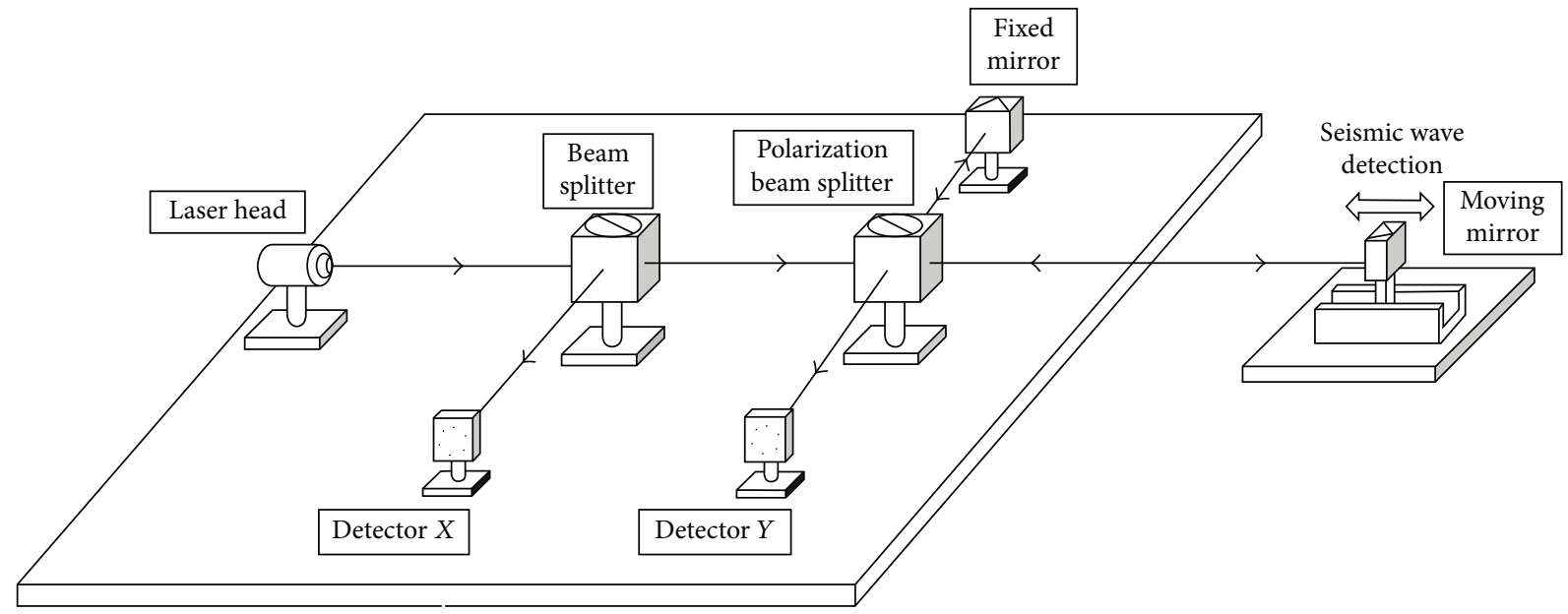

Figure 1: Seismic measurement system based on heterodyne laser interferometer.

particle filter and hidden Markov model. This enables obtaining the real-time phase estimate and the quantified frequency content of seismic data.

In this paper, we propose an interferometric seismometer that is based on a heterodyne laser interferometer and analyze seismic data in the frequency range to estimate epicenter location. Using the orthogonal characteristic of two beams from a laser head, the seismic signals are expressed as a form of intensity signal, which is represented as a trigonometrical function. Hence, the seismic signal can be detected using the laser interferometer. Each observatory has a different experimental environment since the observatory needs to be located in various regions for the seismic signal measurement. The nonuniformity of measurement environment such as temperature and humidity causes the measurement inaccuracy. To compensate for the environmental error that resides in seismic measurement data using a data fusion method, we measure the displacement with two independent laser heads set separately in opposite directions. Using the neural network, the standard deviation of errors is updated adaptively according to the experiment temperature change. With the measured data sets, the environmental error is reduced by projecting the measured data onto the points on the constraint set based on the ideal relationship between the two interferometers $[5,6]$. The compensated data are converted into a signal in the frequency domain to extract the $\mathrm{P}$ - and S-wave arrival times, which are used to calculate the STA/LTA ratio. The difference between the two arrival times leads to the determination of the epicenter location.

\section{A Heterodyne Laser Interferometric Seismometer}

To measure displacement, a heterodyne laser interferometer uses the phase difference caused by the Doppler effect. The heterodyne laser interferometer system consists of a He- $\mathrm{Ne}$ laser head, a beam splitter, a polarization beam splitter, photodetectors, a moving mirror, and a fixed mirror. Figure 1 shows the heterodyne laser interferometer seismic system. The electric fields are measured by detector $X$. Likewise, the electric fields reflected by the fixed mirror and the moving mirror are measured by detector $Y$. The intensities obtained by detectors $X$ and $Y$ are expressed as follows.

$$
\begin{aligned}
I_{\mathrm{r}} & \propto \frac{1}{2}\left(A^{2}+B^{2}\right)+A B \cos \left[\Delta \omega t+\left(\phi_{B}-\phi_{A}\right)\right], \\
I_{\mathrm{m}} & \propto \frac{1}{2}\left(A^{2}+B^{2}\right)+A B \cos \left[\Delta \omega t+\left(\phi_{B}-\phi_{A}\right)+\Delta \phi\right] .
\end{aligned}
$$

In (1), $A$ and $B$ are the magnitudes of the electric fields and $\phi_{A} \phi_{B}$ are the initial phases of laser sources. The signal $I_{\mathrm{r}}$ measured by detector $X$ is the reference intensity, and the signal $I_{\mathrm{m}}$ obtained by detector $Y$ is the measurement intensity. $\Delta \omega$ is the difference between the frequencies of the two orthogonal beams $\omega_{1}$ and $\omega_{2}$, and $\Delta \phi$ is the phase difference caused by the Doppler effect. We use a high-pass filter to eliminate DC components of the intensity signal $I_{\mathrm{m}}$. The initial phase values $\phi_{A}$ and $\phi_{B}$ are ignored to obtain phase information. We obtain the AC signal of $I_{\mathrm{m}}$ as follows.

$$
I_{\mathrm{m}, \mathrm{AC}} \propto A B \cos (\Delta \omega t+\Delta \phi) .
$$

To obtain the phase value, we use a lock-in amplifier. The phase value is derived by applying the $I_{\mathrm{r}}$ and $I_{\mathrm{m}, \mathrm{AC}}$ signals into a power divider, multiplier, and low pass filter, sequentially. As a result, two orthogonal intensity signals of $I_{\mathrm{x}} \propto$ $I_{\mathrm{m}, \mathrm{AC}} \cdot I_{\mathrm{r}}$ and $I_{\mathrm{y}} \propto I_{\mathrm{m}, \mathrm{AC}} \cdot I_{r} e^{j \pi / 2}$ can be represented as a trigonometric function.

$$
\begin{aligned}
& I_{\mathrm{x}} \propto \frac{A B}{2} \cos (\Delta \phi), \\
& I_{\mathrm{y}} \propto \frac{A B}{2} \sin (\Delta \phi) .
\end{aligned}
$$

By using an inverse trigonometric function, the phase information $\Delta \phi$ related to the displacement is obtained. We calculate the displacement change using $\Delta \phi=4 \pi \rho \Delta L / \lambda . \rho$ is the refractive index, $\lambda$ is the average wavelength, and $\Delta L$ is the difference in optical path length between the fixed mirror and the moving mirror. 


\section{Data Fusion-Based Error Compensation}

The environmental error causes a significant problem of a measurement accuracy in case of the nanoscale instrument [7]. In order to improve measurement precision, we use a data fusion method to reduce the error that exists in the measurement data. Data fusion uses two measurement data sets, which in this study are obtained by two laser systems installed separately in opposite directions. To utilize this measured data as a seismic signal, we compensate the error to improve the interferometer's accuracy [8].

We use two independent laser heads, which have the same performance characteristics, for the data fusion method. The two separate laser interferometers measure the same moving mirror with a displacement of $\hat{d}_{1}$ and $\hat{d}_{2}$, respectively. In the ideal case, the displacement $d_{1}$ and $d_{2}$ should satisfy the following constraint, because the two laser interferometers measure the identical moving mirror in the opposite direction. When we measure the displacement, the data includes environmental errors since environmental conditions are not uniform. Therefore, the measurement values $\left(\hat{d}_{1}, \hat{d}_{2}\right)$ do not satisfy the ideal constraint of $d_{1}(k)+d_{2}(k)=0$.

We can represent the relationship between $\widehat{d}_{1}$ and $\widehat{d}_{2}$ in the Cartesian coordinate, where $\widehat{d}_{1}$ and $\widehat{d}_{2}$ are distributed around the constraint of $d_{1}(k)+d_{2}(k)=0$. These patterns show that the measured displacement data contains environmental errors. We project the measured points in the Cartesian coordinate onto the points on the ideal constraint line to compensate for the environmental errors. An objective error function is utilized to minimize the environmental error.

$$
J_{\mathrm{e}}=\frac{1}{2} \sum_{k=1}^{N}\left\|\left(I_{\mathrm{x}}(k)-I_{\mathrm{x}}^{*}(k)\right)^{2}+\left(I_{\mathrm{y}}(k)-I_{\mathrm{y}}^{*}(k)\right)^{2}\right\| .
$$

Here, $I_{\mathrm{x}}$ and $I_{\mathrm{y}}$ are measurement signals that contain environmental error, while $I_{\mathrm{x}}^{*}$ and $I_{\mathrm{y}}^{*}$ are reference signals without environmental error.

Even though the standard deviation is calculated for each measurement step, a compensation parameter $(\varsigma)$ represents one of the values derived under the fixed environment. To improve measurement accuracy, it is necessary to adaptively modify $\varsigma$ by considering temperature change, which is one of the primary environmental error factors. To estimate the adaptive standard deviations, a multilayer neural network is used. The network is composed of one input layer, two hidden layers, and one output layer. The neurons in the hidden layers and the output layer follow the hyperbolic tangent-sigmoid function $f_{1}(x)$ and the log-sigmoid function $f_{2}(x)$ as an activation function, respectively. The activation functions enable the neural network to obtain a complex nonlinear model.

$$
\begin{aligned}
& f_{1}(x)=\frac{e^{x}-e^{-x}}{e^{x}+e^{-x}}, \\
& f_{2}(x)=\frac{1}{1+e^{-x}} .
\end{aligned}
$$

The backpropagation algorithm is applied to train and update weights in the network. The steepest gradient descent method is used during the training period. As a target vector, we obtain the error between the reference signals and the measurement signals.

$$
\mathbf{V}_{\mathrm{T}}=\left.\left[\begin{array}{c}
I_{\mathrm{x}}-I_{\mathrm{x}}^{*} \\
I_{\mathrm{y}}-I_{\mathrm{y}}^{*}
\end{array}\right]\right|_{I_{\mathrm{tp}}(k)=\tau} .
$$

Here, $I_{\text {tp }}(k)=\tau$ is the temperature value during the measurement process. The intensities from (3) are used as the inputs of the neural network during the training period. We define $\mathbf{V}_{\mathrm{I}}=\left[I_{\mathrm{x}}, I_{\mathrm{y}}, I_{\mathrm{tp}}\right]^{T}$ as an input vector and $\mathbf{V}_{\mathrm{O}}=\left[e_{I_{\mathrm{x}}}, e_{I_{\mathrm{y}}}\right]^{T}$ as an output vector. The objective function uses the errors between the output from the neural network and the target vector, $\mathbf{V}_{\mathrm{T}}$ :

$$
E=\frac{1}{2} \sum_{k=1}^{N}\left(\mathbf{V}_{\mathrm{T}}(k)-\mathbf{V}_{\mathrm{O}}(k)\right)^{2},
$$

where $m$ is the amount of sampled data. Weight values are calculated with a steepest gradient descent method and updated until minimizing the objective function.

$$
w_{j}^{k+1}=w_{j}^{k}-\eta \frac{\partial E}{\partial w} .
$$

Here, $\eta$ is a learning rate and $-\partial E / \partial w$ is calculated using the chain rule. To guarantee a reliable performance, the error bound of the objective function is set as $10^{-9}$. The maximum number of the training iterations is limited to 10,000 times for computational efficiency. Finally, we get the neural network modeled in Figure 2.

The estimated error vector $\mathbf{e}_{\mathrm{NN}}=\left[e_{I_{\mathrm{x}}}, e_{I_{\mathrm{y}}}\right]^{T}$ associated with temperature is obtained from the neural network by applying measurement signals $\left(I_{\mathrm{x}}, I_{\mathrm{y}}\right)$ and temperature $\left(I_{\mathrm{tp}}\right)$ as an input vector.

$$
\left[e_{I_{\mathrm{x}}}, e_{I_{\mathrm{y}}}\right]^{T}=\mathrm{NN}\left(I_{\mathrm{x}}, I_{\mathrm{y}}, I_{\mathrm{tp}}\right) .
$$

The adaptive standard deviations $\left(\sigma_{1}^{*}, \sigma_{2}^{*}\right)$ are calculated for each measurement step with the neural network output, $\left(e_{I_{\mathrm{x}}}, e_{I_{\mathrm{y}}}\right)$. The loci of the ellipsoidal equation with a centroid, $\left(\widehat{d}_{1}(k), \hat{d}_{2}(k)\right)$, and the contact points between $x_{m 2}+x_{m 1}=0$ and each ellipse can be represented as follows:

$$
\begin{aligned}
\frac{\left(x_{m 1}-\widehat{d}_{1}(k)\right)^{2}}{\sigma_{1}^{*}(k)^{2}}+\frac{\left(x_{m 2}-\widehat{d}_{2}(k)\right)^{2}}{\sigma_{2}^{*}(k)^{2}} & =1, \\
d_{1}^{*}(k) & = \pm \frac{\zeta_{k}^{2} \widehat{d}_{1}(k)-\widehat{d}_{2}(k)}{1+\zeta_{k}^{2}}, \\
d_{2}^{*}(k) & =\mp \frac{\zeta_{k}^{2} \widehat{d}_{1}(k)-\widehat{d}_{2}(k)}{1+\zeta_{k}^{2}} .
\end{aligned}
$$




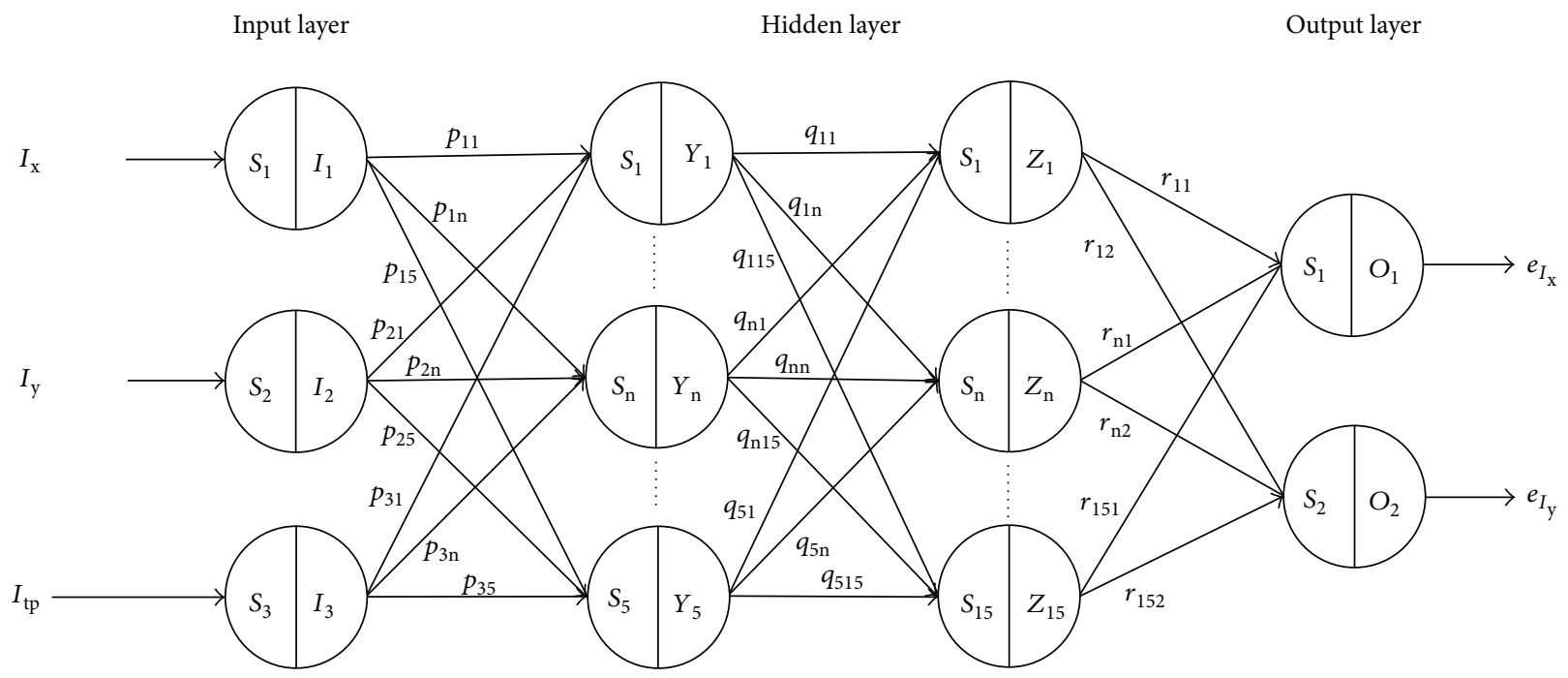

FIGURE 2: Neural network model to compensate for the environmental error.

Here, $\zeta_{k}$ is the adaptive standard deviation ratio, $\zeta_{k}=\sigma_{2}^{*}(k) / \sigma_{1}^{*}(k)$. Finally, the environmental error is compensated by using the adaptive data fusion based on an artificial intelligence projection method.

\section{Seismic Data Analysis and STA/ LTA Algorithm}

To detect the seismic wave, we use the heterodyne laser interferometer system. Using the above equations, we eliminate the environmental error by the data fusion method, enabling more precise measurement of the seismic wave. With the seismic data, we obtain P-S wave arrival time (P-S time), which is an important factor for calculating the epicenter location. The methods that measure P-S time generally analyze seismic data in the time domain. However, if the amplitude of a seismic wave does not change enough during the measurement interval, it is difficult to distinguish between $\mathrm{P}$-wave and S-wave arrival [9]. Hence, we suggest a P-S time measurement method in the frequency domain to more accurately identify the arrival times of P-wave and S-waves.

When we detect the seismic wave with a laser interferometer, the seismic signal can be expressed in terms of the intensity of the laser signal in a trigonometric function. The equation can be represented as follows:

$$
\begin{aligned}
& \widehat{I}_{\mathrm{x}} \propto \frac{A B}{2} \cos (\Delta \widehat{\phi}), \\
& \widehat{I}_{\mathrm{y}} \propto \frac{A B}{2} \sin (\Delta \widehat{\phi}) .
\end{aligned}
$$

In (11), $\widehat{I}_{\mathrm{x}}$ and $\widehat{I}_{\mathrm{y}}$ are the intensity signals after applying the data fusion method to $I_{\mathrm{x}}$ and $I_{\mathrm{y}}$ in (3). As the refractive index $n$ and wavelength $\lambda$ are constants, the change in seismic wave movement, $\Delta L$, is proportional to the phase change, $\Delta \widehat{\phi}$. Therefore, the frequency data of the phase signal $\Delta \widehat{\phi}$ is obtained by the polarity change of the seismic signal, and the $I_{\mathrm{x}}$ and $I_{\mathrm{y}}$ signals are both suitable for frequency analysis. However, the $I_{\mathrm{y}}$ signal is used to analyze the seismic wave as a sinusoidal wave to avoid a latent effect of nonlinearity [10]. The signals are measured in a discrete form. The frequency can be calculated from the signal's polarity change with the following equation.

$$
\operatorname{sgn}\left[\widehat{I}_{\mathrm{y}}(k)\right] \cdot \operatorname{sgn}\left[\widehat{I}_{\mathrm{y}}(k+1)\right]<0 .
$$

Here, $\operatorname{sgn}(\cdot)$ is the signum function. The final frequency value is represented as $f_{c}(k)=N_{c}(k) / 2 . f_{c}(k)$ is the frequency of the incoming intensity signal, and $N_{\mathrm{c}}(k)$ is the number of $\widehat{I}_{\mathrm{y}}(k)$ sign changes in one second. Using this method, we can measure the seismic signal in the frequency domain.

An earthquake's epicenter can be determined using the frequency variation of $\widehat{I}_{\mathrm{y}}(k)$ measured by the heterodyne laser interferometer. We analyze this data with the STA/ LTA ratio to calculate P-S time. With a threshold trigger algorithm, we can enhance the analyzing accuracy of seismic data. The STA/LTA algorithm parameters, such as shorttime window size, long-time window size, and threshold value, are determined by the seismic system environment. The STA/LTA algorithm analyzes the data using the difference between STA and LTA characteristics. Finally, we compare the measured STA/LTA ratio with the threshold value. The accuracy of the analysis data depends on the STA and LTA window sizes and threshold value. The STA and LTA are represented as follows [11-13].

$$
\begin{aligned}
& S_{\mathrm{v}}(n)=\frac{1}{\tau_{\mathrm{s}}} \sum_{k=n-\tau_{\mathrm{s}}}^{n}\left|f_{\mathrm{c}}(k)\right|, \\
& L_{\mathrm{v}}(n)=\frac{1}{\tau_{1}} \sum_{k=n-\tau_{1}}^{n}\left|f_{\mathrm{c}}(k)\right| .
\end{aligned}
$$

Here, $\tau_{\mathrm{s}}$ and $\tau_{1}$ are the size of the short- and long-time windows, respectively. We measure the time when the 


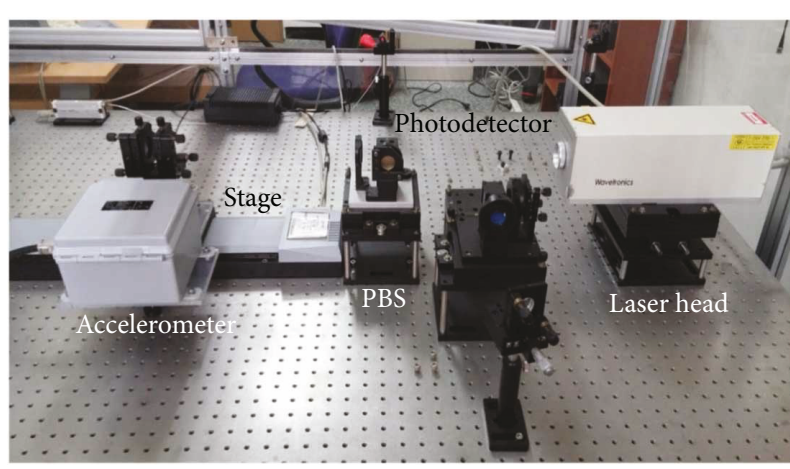

Figure 3: Laser interferometric seismometer.

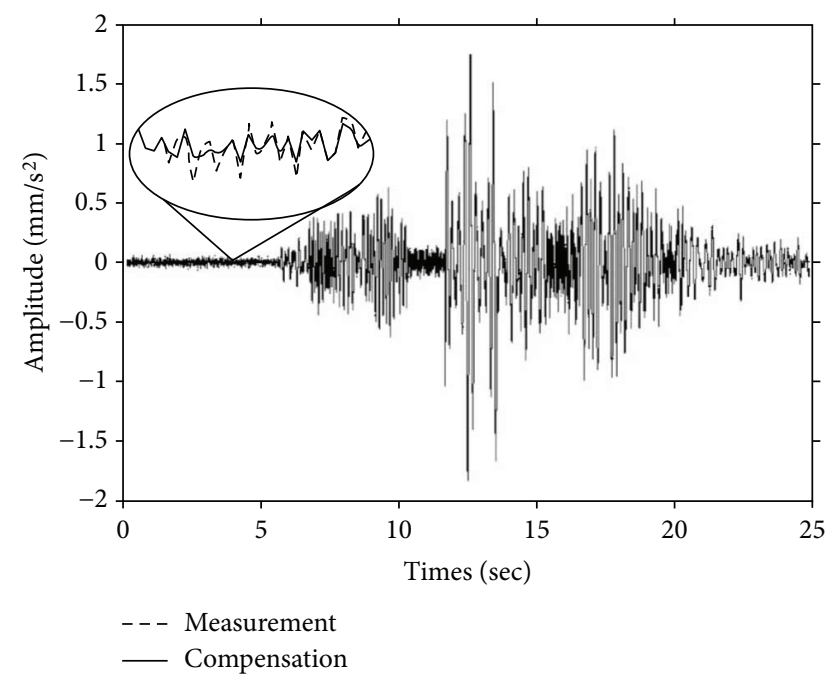

Figure 4: Seismic data compensation with data fusion.

STA/LTA ratio becomes greater than the threshold value. The distance between a seismological observatory and an epicenter is calculated using measured P-S time by the following equation.

$$
D=\frac{V_{\mathrm{P}} V_{\mathrm{S}}}{V_{\mathrm{P}}-V_{\mathrm{S}}} T_{\mathrm{ps}} .
$$

Here, $D$ is the epicentral distance, $V_{\mathrm{p}}$ is the $\mathrm{P}$-wave velocity, $V_{\mathrm{S}}$ is the $\mathrm{S}$-wave velocity, and $T_{\mathrm{ps}}$ is the $\mathrm{P}-\mathrm{S}$ time.

\section{Performance Evaluation}

In this section, we show the performance of the proposed seismic detection system based on a heterodyne laser interferometer. To prove the performance and effectiveness of the interferometric seismometer, we use measured seismic data detected by the laser interferometric seismometer and the accelerometer, respectively. We use the $\mathrm{He}-\mathrm{Ne}$ laser head with an intermode beat of $632.9912 \mathrm{~nm}$ (Wavetronics: WT307B). The laser head emits two-frequency beams of $\lambda_{1}=632.9912576 \mathrm{~nm}$ for vertical polarization and $\lambda_{2}=632.9912604 \mathrm{~nm}$ for horizontal polarization, respectively. The linear stage (PI: M531.5IM) is used as a vibrational source to generate a seismic signal. An accelerometer

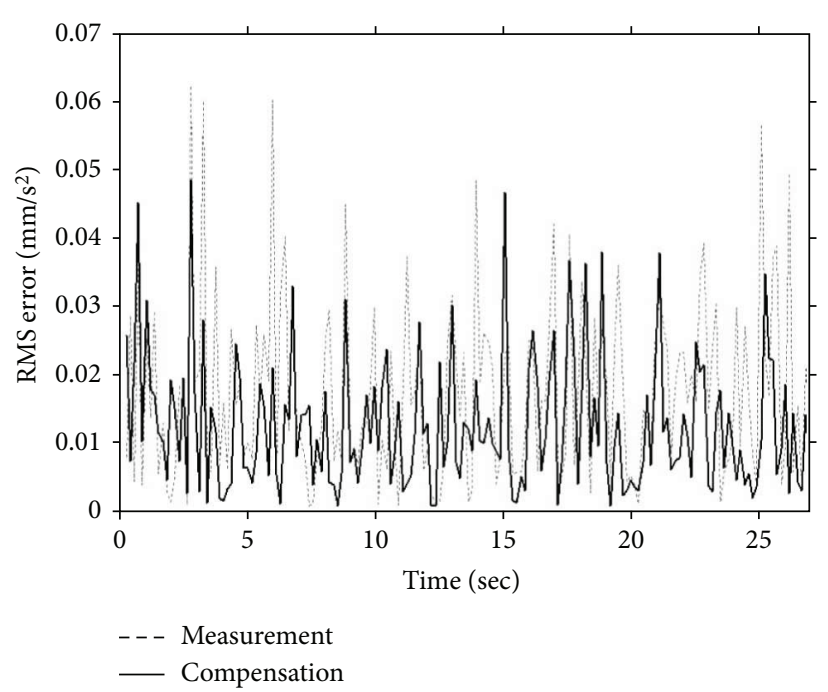

Figure 5: RMS of displacement error.

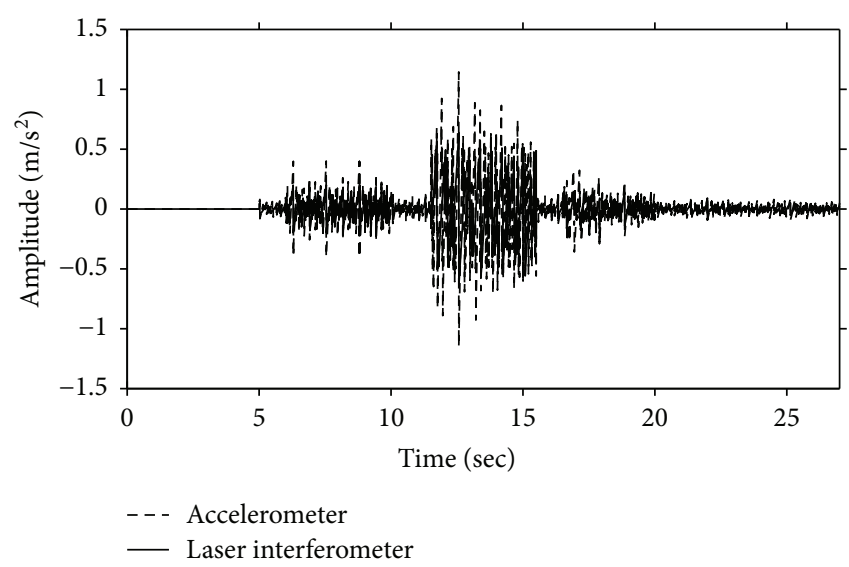

Figure 6: Seismic data detection by interferometric seismometer and accelerometer.

(Mitutoyo: JEP-8A3) is used as a reference. Figure 3 shows the seismic wave measurement system.

First, we prove the environmental error compensation by data fusion method for the measurement precision enhancement in a laser interferometer. In our simulation, we set the wavelength $\lambda$ as $632.9912 \mathrm{~nm}$ and the refractive index as 1.00000026653516. Figure 4 shows the compensated data by the data fusion algorithm. The thick line and thin line represent the compensated seismic data and the measurement seismic data, respectively. To verify the accuracy of compensated seismic data using the data fusion in Figure 4, we compare the root-mean-square (RMS) of the compensated measurement error with the uncompensated one. The RMS error comparison results appear in Figure 5. The environmental errors that occurred in the seismic signal detection are compensated by data fusion. Figure 6 shows the seismic data measured by the laser interferometric seismometer and the accelerometer, respectively. Figure 7 denotes the difference between the true amplitude and the measurement value at each sampling time. The thick solid line represents the RMS error of the 


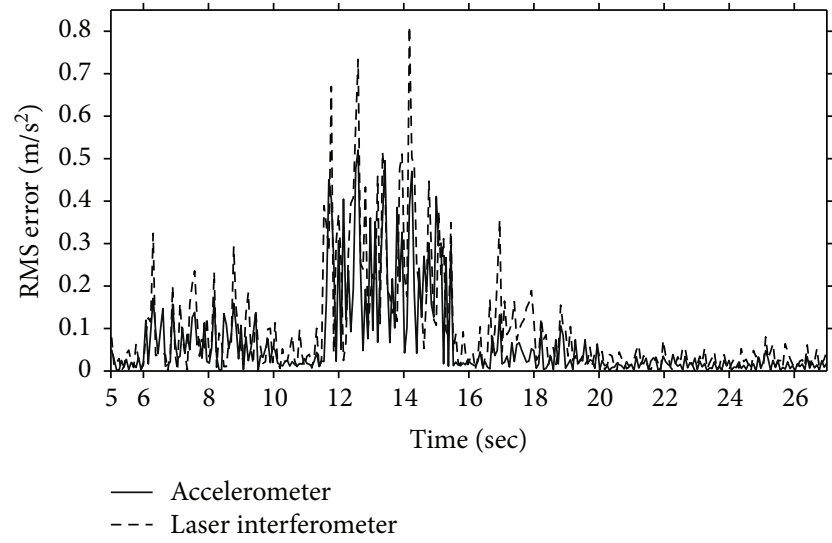

FIGURE 7: RMS error comparison between a laser interferometer and accelerometer.
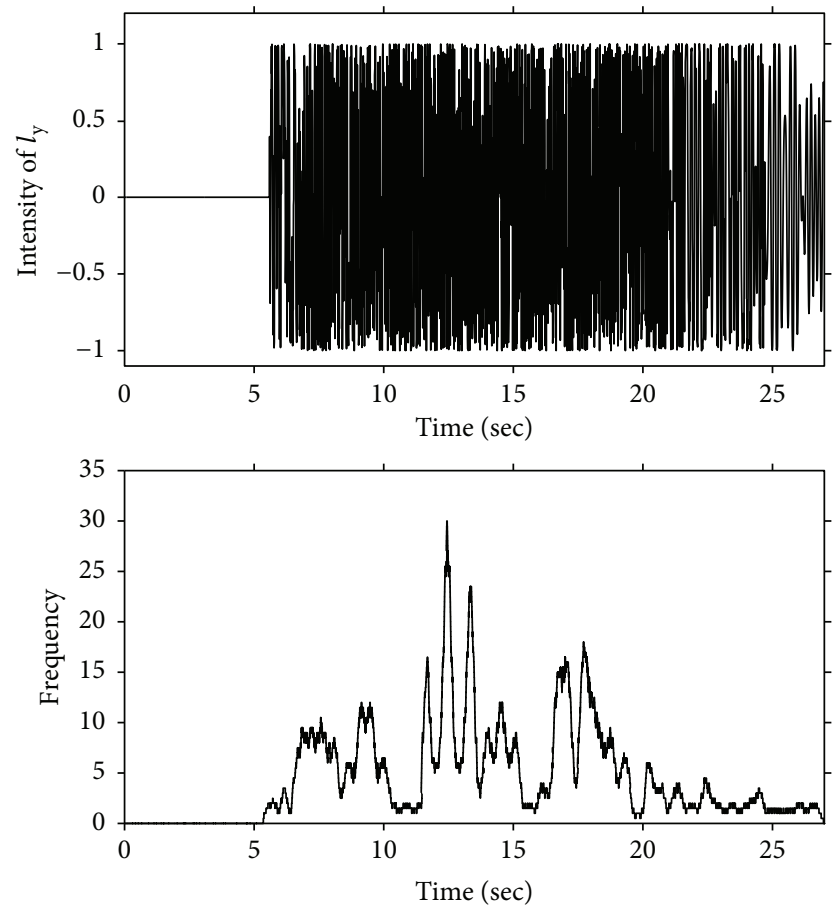

FIGURE 8: Intensity and frequency variation of the seismic signal.

compensated interferometric seismometer amplitude data. The thin dotted line means the RMS error measured from an accelerometer. As shown in this figure, the proposed neural network-based compensated seismic wave is more precise than the measurement of accelerometer.

Figure 8 shows the intensity signal $I_{\mathrm{y}}(k)$ of the seismic wave and the signal in a time-frequency plane measured by the interferometric seismometer. We obtain the intensity signal $\left(I_{\mathrm{y}}\right)$ to minimize the nonlinearity error. The displacement change of the seismic wave is measured from the phase difference between $I_{\mathrm{r}}$ and $I_{\mathrm{m}}$ of the heterodyne interferometer. To analyze the seismic data, we represent the seismic signal in a time-frequency plane and observe sudden frequency changes around 6 and 12 seconds, which are generated by P-wave and $\mathrm{S}$-wave, respectively.

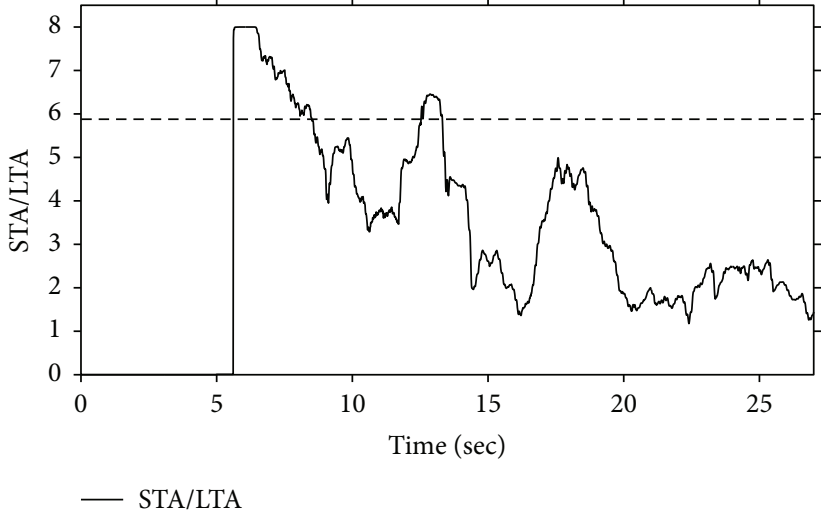

FIgURE 9: STA/LTA ratio.

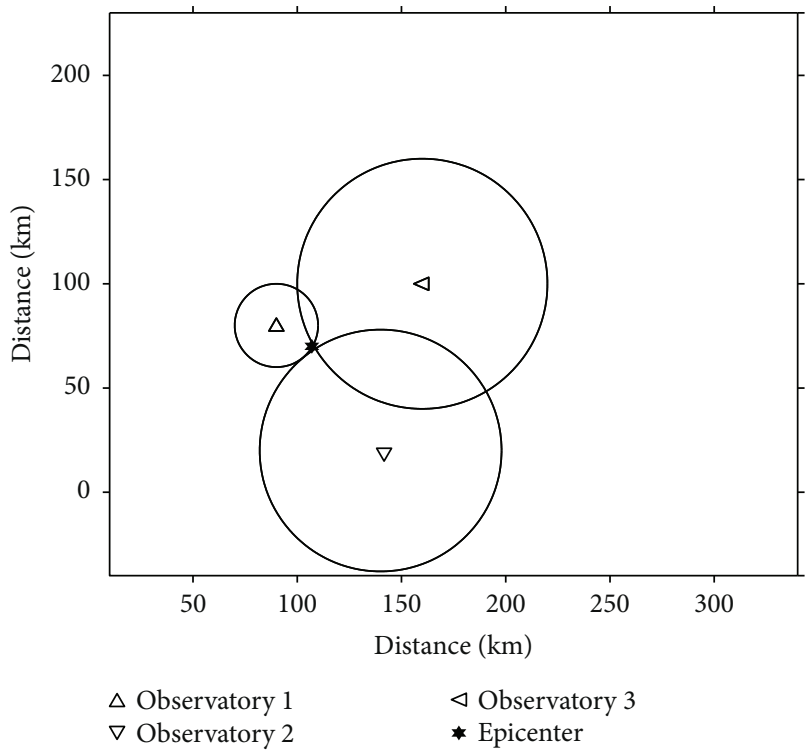

Figure 10: Epicenter location.

We use the STA/LTA algorithm to measure P-S time for calculating the epicenter location. First, we determine the STA and LTA window size. The LTA window size is set as 14 times the STA. Next, the threshold value used for the $\mathrm{P}$-wave and S-wave arrival time is set as 1.3 times the average of the STA/LTA ratio. The STA/LTA ratio and the threshold value are shown in Figure 9.

From the STA/LTA ratio in Figure 8, we measure P-wave and S-wave arrival time as 5.66 and 12.71 seconds, respectively. With the $\mathrm{P}$-wave velocity and the S-wave velocity as $8 \mathrm{~km} / \mathrm{s}$ and $4 \mathrm{~km} / \mathrm{s}$, respectively, we calculate the epicentral distance as $24.20 \mathrm{~km}$ using (14). Figure 10 shows the epicenter location. $n_{\mathrm{e}}$ means the maximum absolute value of the environmental noises. We suppose the parameter $n_{\mathrm{e}}$ as $0.05 \mathrm{~m} / \mathrm{s}^{2}$ in Figure 10. The distance from the observatory to the epicenter is measured by the STA/LTA algorithm.

With three distinct sets of observatory data, we can estimate the epicenter location in the overlapping region. Table 1 shows the RMS error comparison of epicenter localization method based on the laser interferometric seismometer and accelerometer. Although the localization accuracy is 
TABLE 1: RMS error comparison for various environmental errors $\left(n_{\mathrm{e}}\right)$.

\begin{tabular}{lccccc}
\hline Measurement & \multicolumn{3}{c}{ RMS error $(\mathrm{km})$} & $n_{\mathrm{e}}=0.15$ & $n_{\mathrm{e}}=0.2$ \\
\hline Laser interferometric seismometer & $n_{\mathrm{e}}=0.05$ & $n_{\mathrm{e}}=0.07$ & $n_{\mathrm{e}}=0.1$ & 7.83 & 7.19 \\
Accelerometer & 2.54 & 2.81 & 4.27 & 7.19 & 9.45 \\
\hline
\end{tabular}

decreased as the parameter $n_{\mathrm{e}}$ increases, we can confirm that the localization result based on the laser interferometric seismometer shows better performance than accelerometer.

\section{Conclusion}

In this paper, we proposed a seismic signal detection method using a heterodyne laser interferometer in the frequency domain. We applied the data fusion algorithm to minimize the objective error function. To prove the performance of the interferometric seismometer, we compared seismic data measured by a laser interferometric seismometer with data detected by an accelerometer. Moreover, we measured the seismic signal data in the frequency range with the laser interferometer. Using the STA/LTA algorithm, the P-S time was derived. The epicentral distance was calculated using the measured P-S time. We confirmed the effectiveness of the laser interferometer-based seismometer through simulation and experiment.

\section{Conflicts of Interest}

The authors declare that they have no conflicts of interest regarding the publication of this paper.

\section{Acknowledgments}

This work was supported by the National Research Foundation of Korea (NRF) grant funded by the Korea government (MSIP) (NRF-2016R1A2B4011369).

\section{References}

[1] O. Colak, T. Destici, S. Ozen, H. Arman, and O. Cerezci, "Detection of P- and S- wave arrival times using the discrete wavelet transform in real seismograms," The Arabian Journal for Science and Engineering, vol. 34, no. 1A, pp. 79-89, 2009.

[2] G. Hloupis and F. Vallianatos, "Wavelet-based rapid estimation of earthquake magnitude oriented to early warning," IEEE Geoscience and Remote Sensing Letters, vol. 10, no. 1, pp. 4347, 2013.

[3] C. Panagiotakis, E. Kokinou, F. Vallianatos, and P. Automatic, "Automatic $P$-phase picking based on local-maxima distribution," IEEE Transactions on Geoscience and Remote Sensing, vol. 46, no. 8, pp. 2280-2287, 2008.

[4] E. Baziw, "Real-time seismic signal enhancement utilizing a hybrid Rao-Blackwellized particle filter and hidden Markov model filter," IEEE Geoscience and Remote Sensing Letters, vol. 2, no. 4, pp. 418-422, 2005.

[5] A. Effler, R. M. S. Schofield, V. V. Frolov et al., "Environmental influences on the LIGO gravitational wave detectors during the 6th science run," Classical and Quantum Gravity, vol. 32, no. 3, article 035017, 2015.

[6] L. Yan, B. Chen, E. Zhang, S. Zhang, and Y. Yang, "Precision measurement of refractive index of air based on laser synthetic wavelength interferometry with Edlén equation estimation," Review of Scientific Instruments, vol. 86, no. 8, article 085111, 2015.

[7] S. G. Gevorgyan, V. S. Gevorgyan, H. G. Shirinyan, G. H. Karapetyan, and A. G. Sarkisyan, "A radically new principle of operation seismic detector of nano-scale vibrations," IEEE Transactions on Applied Superconductivity, vol. 17, no. 2, pp. 629-632, 2007.

[8] W. Lee, J. Lee, and K. You, "Improved measurement accuracy in heterodyne laser interferometer using WDF," Electronics Letters, vol. 45, no. 21, pp. 1085-1087, 2009.

[9] S. Gabarda and G. Cristobal, "Detection of events in seismic time series by time-frequency methods," IET Signal Processing, vol. 4, no. 4, pp. 413-420, 2010.

[10] W. Hou, "Optical parts and the nonlinearity in heterodyne interferometers," Precision Engineering, vol. 30, no. 3, pp. 337-346, 2006.

[11] B. Sharma, A. Kumar, and V. Murthy, "Evaluation of seismic events detection algorithms," Journal of the Geological Society of India, vol. 75, no. 3, pp. 533-538, 2010.

[12] A. Velasco, S. Hernandez, T. Parsons, and K. Pankow, "Global ubiquity of dynamic earthquake triggering," Nature Geoscience, vol. 1, no. 6, pp. 375-379, 2008.

[13] A. Maggi, C. Tape, M. Chen, D. Chao, and J. Tromp, “An automated time-window selection algorithm for seismic tomography," Geophysical Journal International, vol. 178, no. 1, pp. 257-281, 2009. 


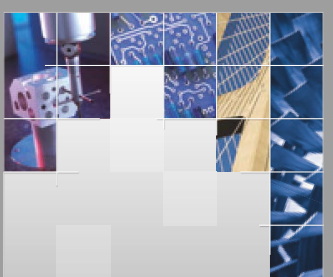

\section{Enfincering}
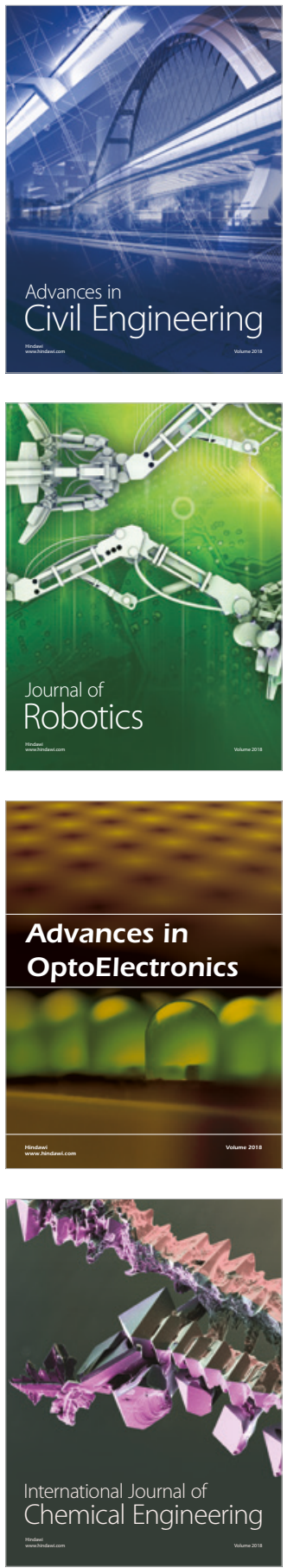

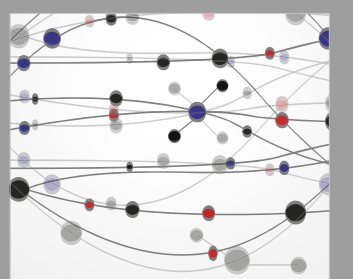

\section{Rotating \\ Machinery}

The Scientific World Journal

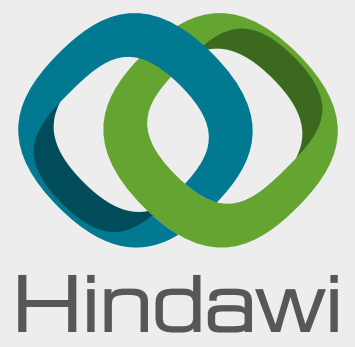

Submit your manuscripts at

www.hindawi.com
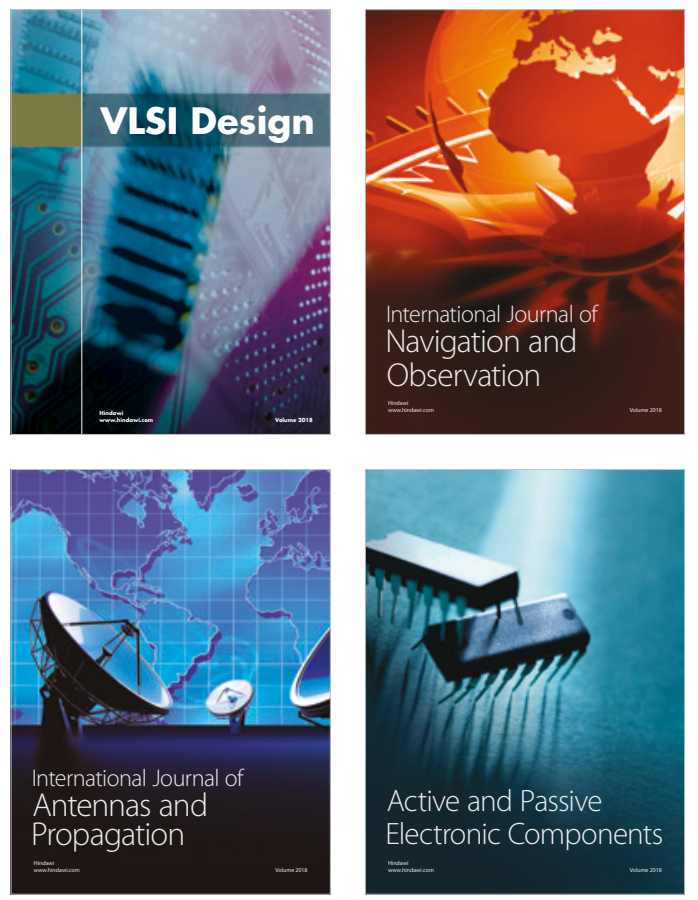
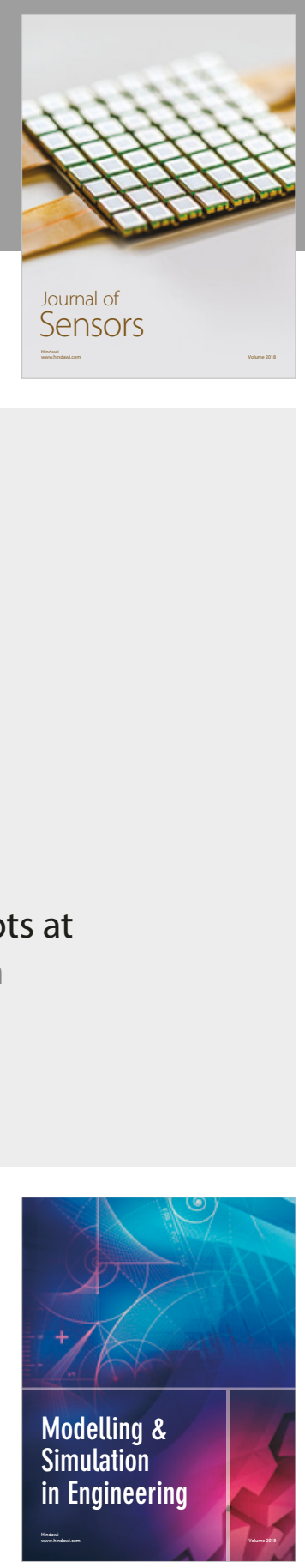

\section{Advances \\ Multimedia}
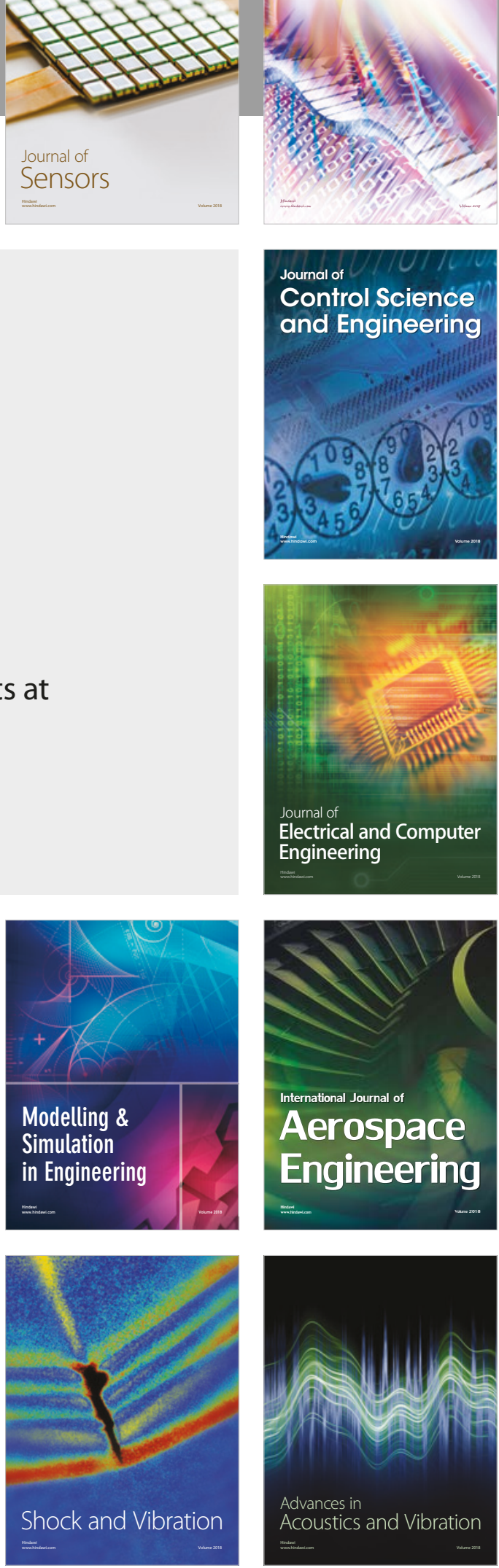